\title{
Del empowerment al outsourcing como estrategia de crecimiento
}

\section{From Empowerment to Outsourcing as a Growing Strategy}

\author{
Víctor Manuel Cornejo Aparicio*
}

http://dx.doi.org/10.21503/CienciayDesarrollo.2006.v7.03

\section{RESUMEN}

Las empresas en la actualidad requieren de la especialización de sus actividades para garantizar la calidad en sus actividades o productos en sus diversas áreas de desarrollo y ámbitos laborales, pero también es cierto que a medida que crecen sus operaciones, se ven empujadas a desarrollar actividades que adquieren tamaños considerables y provocan desgaste del aparato administrativo y consumo de recursos empresariales, lo que en definitiva merma y aletarga el avance de estas empresas. Por ello, un plan bien definido para desarrollar empowerment en la empresa debería garantizar la calidad con que espera desarrollar y, en un futuro próximo, independizar ciertas áreas funcionales que ayuden a su formación como empresa especializada. Esto le permitiría recuperar recursos y, posteriormente, destinarlos a áreas o actividades que redunden en el crecimiento de la empresa, configurando así una estrategia de crecimiento en escalas cada vez mayores.

Palabras clave: empowerment, outsourcing, estrategia empresarial.

\section{ABSTRACT}

Companies nowadays require the expertise of their activities to ensure quality in their activities and / or products in various development areas and fields of work, but it is also true that as their operations grow, their activities also acquire a considerable wear off from the administrative part as well as the enterprise resources, which reduce the progress that these companies are trying to achieve, this is why a plan to develop well-defined Empowerment in the company, ensure the quality with which the company thinks to develop, and in the near future, independent certain functional areas to form specialized companies, which would allow to get resources and then put them to areas or activities that would return more interest in the rate of growth of the company, this would mean a growing strategy in a bigger scale.

Key words: empowerment, outsourcing, business strategy.

\footnotetext{
* Universidad Alas Peruanas.
} 


\section{INTRODUCCIÓN}

\section{Empowerment}

\section{Definiciones de empowerment}

Empowerment quiere decir potenciación o empoderamiento, que es el hecho de delegar poder y autoridad a los subordinados y de conferirles el sentimiento de que son dueños de su propio trabajo (1).

Es un proceso estratégico que busca una relación de socios entre la organización y su gente, aumentar la confianza, la responsabilidad, la autoridad y el compromiso de servir mejor al cliente (2).

Como conclusión, el empowerment (o la gestión moderna de personas y capital humano) aporta ventajas comparativas a la compañía en términos de relaciones con los clientes, costes, agilidad y flexibilidad... rentabilidad en una palabra... aunque la transición no sea nada sencilla (3).

El empowerment busca que los beneficios óptimos de la tecnología de la información sean alcanzados. Los miembros, equipos de trabajo y la organización tendrán completo acceso y uso de información crítica, poseerán la tecnología, habilidades, responsabilidad, y autoridad para utilizar la información y llevar a cabo el negocio de la organización (4).

El empowerment permite al recurso humano asumir mayores responsabilidades y autoridad mediante la formación, la confianza y el apoyo emocional, y por ello está vinculado directamente con la toma de decisiones. Se entiende que la toma decisiones no es un proceso solamente lógico, sino también psicológico, y depende de la reunión y el análisis lógico de la información, de las características de los miembros tales como sus motivos, valores y percepciones, de las características de grupo tales como normas y estilos de liderazgo y de las características de la organización tales como la competencia de grupos y las pautas de comunicación (5).

Empowerment es dar y adquirir en forma continua y permanente el poder del conocimiento. No se trata de poder político o administrativo, sino de "conocimiento", lo que es característico, intrínseco y propio de la sociedad en que ya vivimos, la sociedad de la información y del conocimiento, la knowledge-based society, que ha reemplazado sin vueltas a la sociedad industrial (6).

\section{Cualidades y caracteristicas del empowerment}

El empowerment se puede otorgar a una persona o a un equipo conformado por grupos de trabajo con empleados responsables de un producto o servicio, y que comparten el liderazgo, colaboran en el mejoramiento del proceso del trabajo, planean y toman decisiones relacionadas con el método de trabajo. Los equipos con empowerment tienen las siguientes características (7):

- Comparten el liderazgo y las tareas administrativas.

- Los miembros tienen facultad para evaluar y mejorar la calidad del desempeño y el proceso de información.

- El equipo proporciona ideas para la estrategia de negocios.

- Son comprometidos, flexibles y creativos.

- Coordinan e intercambian con otros equipos y organizaciones.

- Mejoran la honestidad, las relaciones con los demás y la confianza.

- Tienen una actitud positiva y son entusiastas. 


\section{Participación del Personaly Desarrollo de Empowerment}

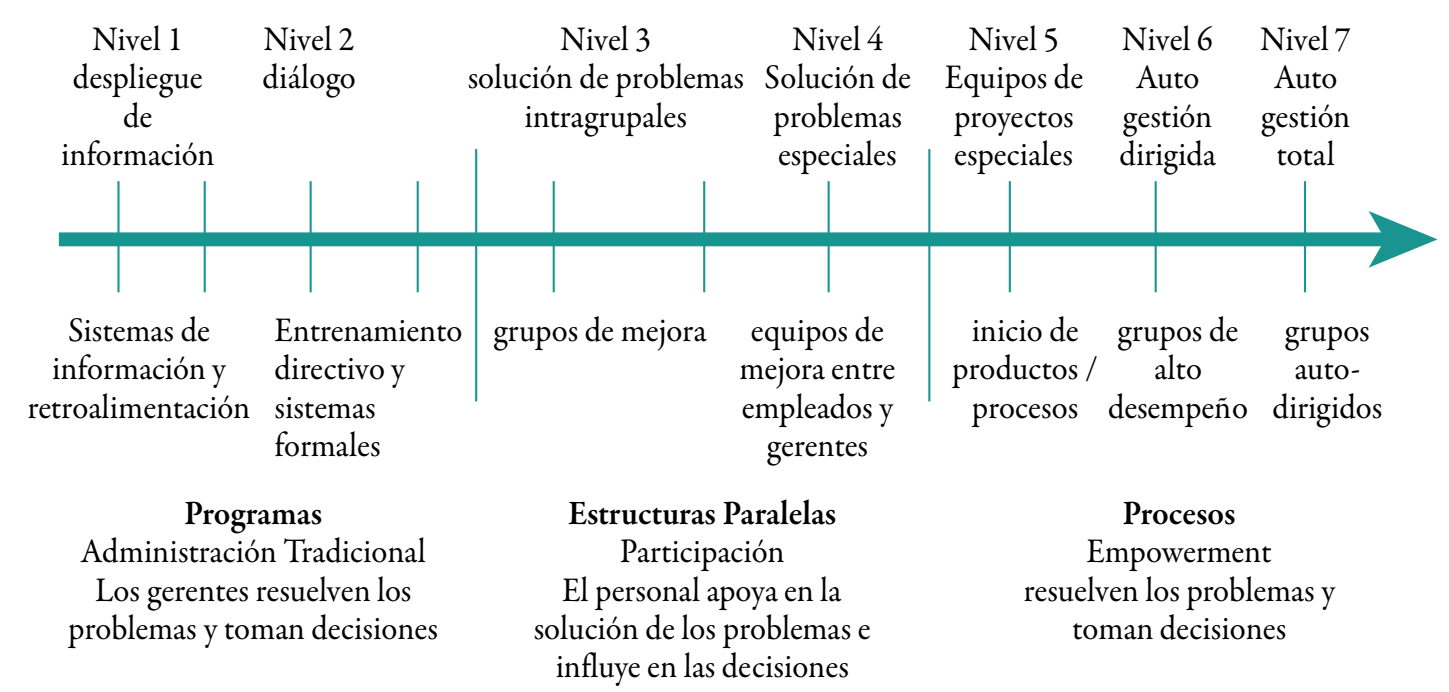

De los factores que dan lugar a que se produzca este cambio, la mayoría de ellos son las fuerzas externas que obligan a la organización a cambiar. Estos son (8):

- Competencia global acelerada.

- Clientes insatisfechos.

- Poca rapidez en la innovación o introducción del producto.

- Organizaciones más planas y lineales.

- Inercia y lucha burocrática.

- Tecnología que cambia rápidamente.
- Cambio de valores en los empleados.

- Estancamiento en la eficiencia o en la productividad.

\section{Técnicas, elementos y métodos del equipo con em- powerment}

El equipo está donde el empowerment crece. El ingrediente más importante del empowerment es la relación directa entre usted y la gente con la que trabaja. Son tres las consideraciones que se deben tener para trabajar con empowerment:

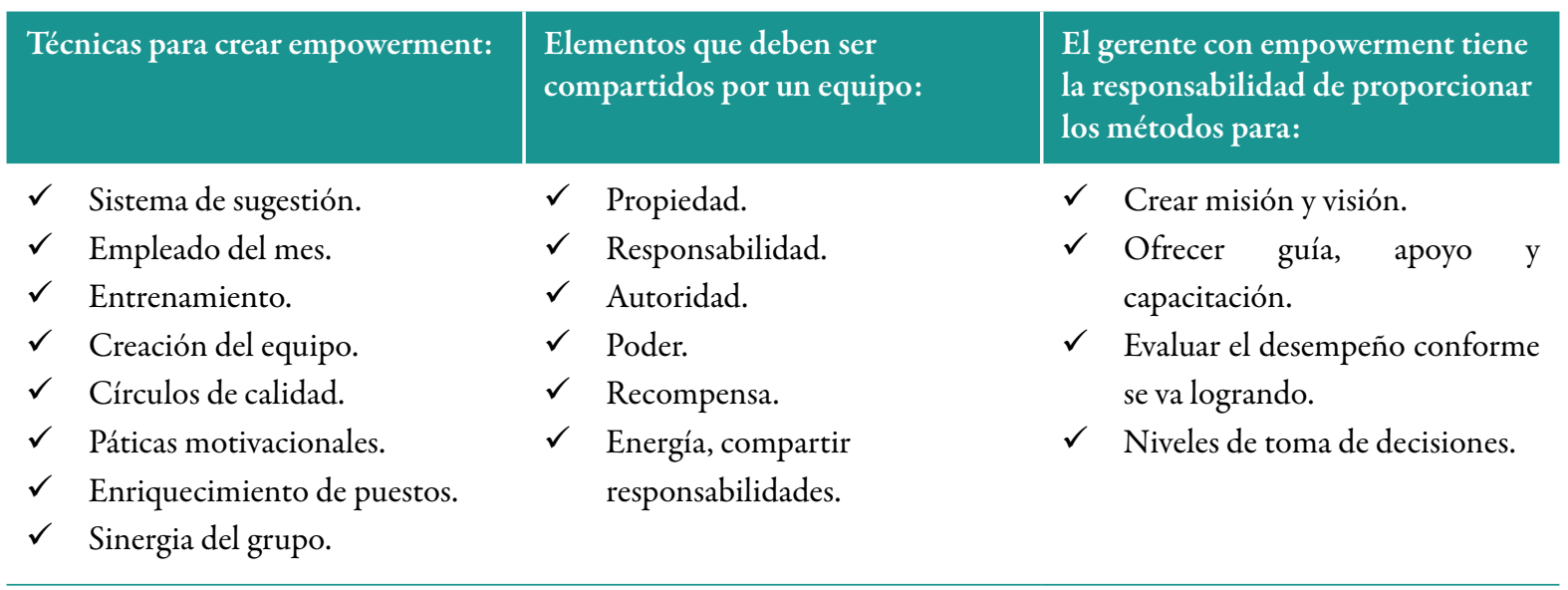

Fuente: www.fundameca.org.mx 
La toma de decisiones es el proceso central del trabajo en equipo. Es importante entender que hay diferentes niveles de participación en el ejercicio de la toma de decisiones. La siguiente escala de decisiones representa el nivel que un gerente puede escoger cuando comparte la toma de decisiones con su equipo.

Escala de decisiones: (Ver figura abajo)

\section{Ventajas del empowerment}

Todos los beneficios redundan en una organización más rentable, gracias a tener clientes más satisfechos y personas más contentas y comprometidas.

A nivel de las personas, con el empleo de este método las personas se sienten competentes, considerándose eficaces y sintiéndose integradas a la organización y alineadas con su estrategia, en la que asumen el nivel más alto de delegación por parte de sus superiores.
Entre las principales ventajas que se obtienen implantando el empowerment en una empresa se tienen las siguientes:

1. Aumenta la satisfacción y confianza de las personas que integran la organización.

3. Aumenta la responsabilidad, autoridad y compromiso en la satisfacción del cliente.

4. Aumenta la creatividad y disminuye la resistencia al cambio.

5. Los integrantes de la organización comparten el liderazgo.

6. Mejora la confianza en la comunicación y las relaciones.

7. Incrementa el entusiasmo y una actitud positiva.

8. Se tiene un proceso más eficiente para la toma de decisiones.

9. Mayor comunicación en todos los niveles y en todas las direcciones.

\section{Nivel cinco: Delegación}

Usted les piede que decidan.

Ellos toman el control.

\section{Nivel cuatro: Colaboración}

Se toma una decisión que les guste a todos.

Cada quien toma completa responsabilidad.

\section{Nivel tres: Diálogo}

Se discuten profundamente los temas antes de que usted decida. Todo el mundo acepta su decisión.

Nivel dos: Cooperación

Pide información antes de tomar una decisóin.

Escucha los comentarios.

Nivel uno: Dirección

Les dice lo que usted decidio.

Les pregunta que piensan acerca de eso.

Fuente: Peter Block. The empowered manager. Positive political skills at work. Jossey-Bass Publishers, San Francisco Oxford, 2001, U.S.A. 


\section{Desventajas del empowerment}

Aunque algunas compañías buscan facultar a su personal a través del empowerment, generalmente fracasan, pues no logran los resultados deseados.

Esto se debe generalmente a que no se le pone la atención debida y porque no se lo muestra de manera concreta, de tal manera que todos sepan de qué se trata y cuáles son los resultados que se esperan de él.

Si no se incorporan los factores fundamentales que toca el empowerment (satisfacción del cliente, mejorar resultados financieros y retener y atraer a los empleados adecuados), los gerentes obtendrán solamente resultados mediocres.

Opciones al delegar responsabilidad:

- Encomendarle el trabajo a la persona adecuada.

- Delegar autoridad para llevar a cabo el trabajo y tomar decisiones.

- Delegar el trabajo sin dar autoridad para la toma de decisiones.

- Hacer uno mismo el trabajo.

La pirámide del poder se basa en:

- Confianza.

- Respeto.

- Permiso para fallar.

Las siguientes acciones definen la estructura de la pirámide del poder:

- Definir responsabilidad.

- Delegar autoridad.

- Definir estándares de desempeño.

- Entrenamiento y desarrollo.
- Brindar información y conocimiento.

- Brindar retroalimentación.

- Reconocimiento.

Se ha de tener en cuenta que para introducir el empowerment dentro de la cultura de la organización hace falta un importante esfuerzo de las personas que la integran, pues el impacto sobre ellas es muy importante.

Se debe tener muy clara la visión del proyecto y los resultados que se conseguirán, ya que el camino no es sencillo. Abandonar el proyecto sin finalizarlo es peor que no haberlo comenzado, debido a la incertidumbre y a la tensión que crea en las personas.

Para tener clara la complejidad del proyecto, sólo se ha de recapacitar en que se debe cambiar lo que llevamos practicando por 20 ó 30 años (organización centralista, autoritarista e individualista, y donde las personas son ejecutoras de órdenes). El objetivo es convertir a la organización en otra totalmente distinta, con un sistema descentralizado, donde las decisiones son compartidas y las personas, trabajando en equipo, toman decisiones en todos los niveles. Si todo esto hay que cambiarlo en algunos meses o años, debemos imaginar el tipo de cambio que hay que realizar.

\section{Conclusiones respecto al empowerment}

Como conclusiones podemos afirmar que las personas que trabajan en la empresa son el motor de la misma, y es preciso por ello aspirar a un crecimiento personal que se amplía al mismo tiempo y que aprovecha las capacidades de cada una de ellas. Este es el momento de concentrarse en la tarea de obtener lo mejor de nuestra gente.

El empowerment es la herramienta que hace posible el cambio, pero, como hemos comenta- 
do, no es fácil de implantar. Se requiere de una cultura organizativa que lo haga posible, con una implicación directa de la Dirección General y la asunción de una definida estrategia empresarial. No hay que olvidar que el activo principal que tiene una organización son las personas, y que debemos potenciarlas para que se sientan implicadas. Sin el clima adecuado, ello no es posible, y por eso se hace necesario que las políticas de gestión de recursos humanos tiendan a fomentar y a promover al personal dentro de la empresa. De este modo, este potencial se conveertirá en un valor estratégico competitivo para la organización.

\section{Outsourcing}

\section{Definiciones de outsourcing}

Outsourcing y externalización son términos empleados indistintamente para definir una herramienta de gestión que designa una área o actividad de trabajo de nuestra organización a otra empresa externa, denominada outsourcer, especializada en este servicio demandado. Charles Handy, defensor de la empresa virtual, define el término outsourcing como la externalización de aquellas funciones que no se consideran competencias nucleares de la organización. Este autor emplea el concepto nuclear como el número de actividades o funciones vitales de la organización (11).

La subcontratación (del inglés outsourcing), también llamada tercerización o externalización, es el proceso económico por el cual una empresa determinada mueve o destina a una empresa externa los recursos orientados a cumplir ciertas tareas, por medio de un contrato. Esto se da especialmente en el caso de la subcontratación de empresas especializadas. Para ello, se puede contratar sólo al personal, en cuyo caso los recursos los aportará el cliente (instalaciones, hardware y software), o contratar tanto el personal como los recursos (12).
Básicamente se trata de una modalidad según la cual determinadas organizaciones, grupos o personas ajenos a la compañía son contratados para hacerse cargo de parte del negocio o de un servicio puntual dentro de ella. La compañía delega la gerencia y la operación de uno de sus procesos o servicios a un prestador externo (outsoucer), con el fin de agilizarlo, optimizar su calidad y/o reducir sus costos (13).

El outsourcing o la tercerización es una herramienta que permite a las empresas enfocarse en hacer lo que realmente hacen bien (14).

Outsourcing es un término que se está aplicando incorrectamente a la contratación de servicios tipo "Man Power" o de proyectos llave en mano. El objetivo es claro, e implica ceder al tercero la responsabilidad y el compromiso del área relacionada, para lo cual la empresa contratada debe posicionarse dentro del organigrama de la empresa contratante (15).

En el outsourcing, se produce la segregación de actividades o departamentos de la empresa a fuentes externas, para que realicen el trabajo bajo condiciones perfectamente especificadas en tiempo de entrega, calidad, costo, además de las garantías y penalizaciones en caso de incumplimiento. El término out significa "fuera", y sourcing, "fuente" (16).

\section{Cualidades y caracteristicas del outsourcing}

Hasta hace un tiempo, esta práctica era considerada como un medio para reducir los costos; sin embargo, en los últimos años ha demostrado ser una herramienta útil para el crecimiento de las empresas por razones tales como:

- Es más económica. Reducción y control del gasto de operación.

- Concentración de los negocios y disposición más apropiada de los fondos de capital, debi- 
do a la reducción o no uso de los mismos en funciones no relacionadas con la razón de ser de la compañía.

- Acceso al dinero efectivo. Se puede incluir la transferencia de los activos del cliente al proveedor.

- Manejo más fácil de las funciones complejas o que están fuera de control.

- Disposición de personal altamente capacitado.

- Mayor eficiencia.

Todo esto permite a la empresa enfocarse ampliamente en asuntos empresariales, tener acceso a capacidades y materiales de clase mundial, acelerar los beneficios de la reingeniería, compartir riesgos y destinar recursos para otros propósitos (207).

\section{Etapas en el proceso de tercerización (18)}

La decisión de tercerizar implica un proceso analítico en cuatro etapas sucesivas (Ronan Mc Ivor, 2000):

Etapa 1. Definir las actividades clave o centrales de la empresa. Las actividades centrales o clave son percibidas por los clientes como aquellas que agregan valor y por lo tanto son básicas para la competitividad de la empresa. Son las que permiten a la empresa diferenciarse de sus competidores, por ejemplo en la calidad de sus productos o servicios, en su capacidad para reconocer las necesidades del mercado e innovar o mantener un bajo nivel de costos. Distinguir entre actividades que son clave pero que podrían ser consideradas para ser tercerizadas y las que son críticas para la competitividad de la empresa y que no deberían tercerizarse es una tarea seria a la que debe dedicarse tiempo y cuidado. Las actividades que son críticas para la empresa deben ser manejadas al interior de la empresa, y son aquellas en las que la empresa debe seguir invirtiendo y construyendo capacidades únicas y diferentes. Definir qué acti- vidades podrían ser consideradas para tercerizar requiere entender muy bien la industria y el negocio, la dinámica del entorno, la competencia y la propia naturaleza de la ventaja de la empresa.

Etapa 2. Evaluar todas las actividades relevantes de la cadena de valor. En esta etapa es necesario aplicar una perspectiva de la cadena de valor de la empresa para identificar y analizar los costos de cada actividad. Se analizan los costos por actividad para evaluar y comparar las economías que podrían generarse en caso de tercerizar alguna de ellas. Contabilidades tradicionales suelen no ser adecuadas para este tipo de análisis, y es necesario utilizar métodos de costeo basados en actividades ( $\mathrm{ABC}$ costing). Con los resultados del análisis y costeo de actividades, se está en condiciones de comparar los costos entre hacer y comprar.

Etapa 3. Comparación de costos entre hacer y comprar. En la etapa anterior se determinaron los costos de .hacer. En esta etapa es necesario determinar el costo de comprar, lo cual no solamente implica considerar el precio del producto o servicio ofrecido por el proveedor sino identificar y medir todas las actividades y costos internos que se le generan a la empresa en caso de tercerizar. Se deberá prestar atención a todos los costos involucrados en el proceso de adquisición y de gestión de la relación con el proveedor por parte de la empresa. Se debe considerar desde la búsqueda inicial y comparación entre proveedores hasta la gestión del día a día de los sistemas de información y logística, como por ejemplo controlar al proveedor, costos de garantías y contratos, costos asociados a cooperar con el proveedor para la mejora del producto o servicio. El costo de tercerizar se compone del precio del producto o servicio más todos los costos de transacción que se generan antes, durante y después de tercerizar. Si el costo de comprar, incluido el costo de gestionar la relación con el proveedor, es más bajo que el costo de hacer, entonces se puede pasar a la siguiente etapa. 
Etapa 4. Análisis de la relación con el proveedor. Tercerizar actividades no periféricas suele requerir una estrecha relación de trabajo con el proveedor, basada en la cooperación mutua y en el intercambio de información. Las asociaciones y alianzas implican riesgos y deben gestionarse, lo cual implica costos adicionales. Ello puede ser más o menos costoso, dependiendo del tipo de industria, de la estructura competitiva de la misma, del poder relativo entre proveedor y cliente, y de los riesgos de que el proveedor pueda asumir una conducta oportunista en el futuro. Cuando una industria se torna más competitiva y asume forma de clúster dinámico, las empresas tienden a especializarse, aumenta la variedad y calidad de proveedores a lo largo de toda la cadena de abastecimiento, y consecuentemente se incrementan las posibilidades de encontrar un proveedor adecuado y confiable. A su vez, el costo de tercerizar se reduce en este tipo de industrias, ya que las transacciones son gobernadas por los mecanismos propios del mercado o por contratos relativamente sencillos. En cir- cunstancias en las que la tercerización requiere de alianzas, se pierden algunas de las ventajas, como por ejemplo la flexibilidad. La decisión de tercerizar o no dependerá de la posibilidad de gobernar la relación con el proveedor bajo algún tipo de mecanismo contractual o asociativo que resulte confiable.

\section{Ventajas del outsourcing}

Se pueden mencionar los siguientes beneficios o ventajas del proceso de outsourcing [207]:

- Los costos de manufactura declinan y la inversión en planta y equipo se reduce.

- Permite a la empresa responder con rapidez a los cambios del entorno.

- Incremento en los puntos fuertes de la empresa.

- Ayuda a construir un valor compartido.

- Ayuda a redefinir la empresa.

\section{Etapa 1: Definir las actividades claves 0 centrales de la empresa

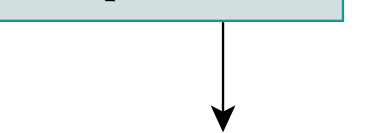

Etapa 4: Análisis de la relación con el proveedor. 
- Construye una larga ventaja competitiva, sostenida mediante un cambio de reglas y un mayor alcance de la organización.

- Incrementa el compromiso hacia un tipo específico de tecnología que permite mejorar el tiempo de entrega y la calidad de la información para las decisiones críticas.

- Permite a la empresa poseer lo mejor de la tecnología sin la necesidad de entrenar personal de la organización para manejarla.

- Permite disponer de servicios de información en forma rápida, considerando las presiones competitivas.

- Aplicación del talento y de los recursos de la organización a las áreas clave.

- Ayuda a enfrentar cambios en las condiciones de los negocios.

- Aumento de la flexibilidad de la organización y disminución de sus costos fijos.

En conclusión, las ventajas del outsourcing o tercerización dependen de un conjunto complejo de factores. La decisión debería estar basada en un cuidadoso análisis estratégico, de los costos de las actividades, de la relación con el proveedor y en una detallada comparación de costos entre hacer y comprar. Dependerán también de la confiabilidad de los proveedores y de la posibilidad de gobernar eficientemente la relación proveedorcliente. Del otro lado del mostrador, las empresas que aspiren a convertirse en proveedoras preferentes de clientes que deciden tercerizar, además de ofrecer un producto o servicio a precio conveniente, deberán dar señales de confiabilidad y aprender a manejar la relación con el cliente de tal forma que no se agreguen costos de transacción innecesarios. Las nuevas tecnologías de información son un elemento que facilitará cada vez más la gestión de las relaciones entre proveedores y clientes, y por lo tanto también facilitarán y promoverán la práctica de la tercerización. La tendencia globalizadora también seguirá facilitando la tercerización fuera de las fronteras nacionales, lo que podría ser una oportunidad para países en desarrollo.

\section{Desventajas del outsourcing}

En todo proceso existen aspectos negativos que forman parte integral del mismo, y el outsourcing no queda exento de esta realidad.

Se pueden mencionar las siguientes desventajas del outsourcing (17):

- Estancamiento en lo referente a la innovación por parte del suplidor externo.

- La empresa pierde contacto con las nuevas tecnologías que ofrecen oportunidades para innovar los productos y procesos.

- Al obtener los conocimientos acerca del producto en cuestión, existe la posibilidad de que el suplidor externo los use para empezar una industria propia y se convierta de suplidor en competidor.

- El costo ahorrado con el uso de outsourcing puede que no sea el esperado.

- Las tarifas incrementan la dificultad de implementar otra vez las actividades que vuelvan a representar una ventaja competitiva para la empresa.

- Alto costo en el cambio de suplidor en caso de que el seleccionado no resulte satisfactorio.

- Reducción de beneficios.

- Pérdida de control sobre la producción.

\section{MATERIAL Y MÉTODOS}

\section{La estrategia de crecimiento basado en el empowerment y el outsourcing}

Las decisiones relacionadas con el outsourcing, en muchas ocasiones, tienen un elevado 
componente estratégico, por lo que la estrategia necesita estar perfectamente definida para tomar las decisiones alineadas con ella (19).

El outsourcing, en cuanto externalización completa de los sistemas y procesos, puede resultar la mejor alternativa, pues recomienda la elaboración de un plan detallado al respecto que garantice el mantenimiento de la inteligencia dentro de la empresa. "De lo contrario, se corre el peligro de perder el valor estratégico de la aplicación TI, el vínculo entre las tecnologías de la información y el negocio. Es necesario que incluso en los grandes proyectos de outsourcing, siga existiendo un departamento interno que garantice el mantenimiento de esa vinculación. Si no es así, dado el potencial de las TI para mejorar la productividad de las empresas, la externalización puede derivar a largo plazo en una peligrosa desventaja competitiva”. (20)

El outsourcing es el proceso por el cual una firma identifica una porción de su negocio que podría ser desempeñada más eficientemente y más efectivamente por otra corporación, la cual es contratada para desarrollar esa porción de negocio. Esto libera a la primera organización y le permite enfocarse en la parte o función central de su negocio (21).

\section{El crecimiento empresarial}

El Enfoque Basado en los Recursos (EBR) propone un modelo integrador que incide sobre los aspectos dinámicos, a partir de una revisión de diferentes trabajos que desde este punto de vista analizan distintos aspectos del proceso de crecimiento empresarial. Se parte de la idea de que cualquier opción estratégica precisa de recursos y genera recursos, de manera que la estrategia ejerce un impacto sobre los recursos de la empresa, aunque a su vez la estrategia desarrollada se ve afectada por los recursos. Partiendo de esta premisa, en el modelo se integran y sistematizan las diferentes decisiones de estrategia corporativa de la empresa que aparecen en la literatura sobre el EBR, con especial énfasis en las estrategias de diversificación. Asimismo, el modelo tiene una serie de implicaciones sobre el análisis basado en aspectos como los límites de la empresa o el entrepreneurship. Del análisis se extrae un conjunto de proposiciones contrastables sobre diferentes aspectos del crecimiento desde el EBR (22).

En el advenimiento de nuevos tiempos, es una realidad que las poblaciones crecen; y con ello, la demanda de los mercados se incrementan, y aparecen nuevos competidores y las empresas ya posesionadas en el mercado se ven en la necesidad de crecer, entre otros aspectos, en tamaño de producción, en capacidad operativa, personal, etc. En razón de esto, las empresas no se pueden sentir ajenas a esta corriente incesante de búsqueda de alta competitividad y optimización de recursos.

\section{La necesidad de recursos}

Una encuesta realizada por la EVCA (European Private Equity and Venture Capital Association): "EVCA 2005b. Fulfilling the Promise of Venture-backed High Potential Companies: Why We Need to Fix Small Cap Markets in Europe. An EVCA High Tech Committee Paper", sobre las PYME que han recibido financiación procedente de fondos de capital de riesgo, destaca las siguientes conclusiones (23):

- El 95\% de las empresas encuestadas reconocieron que no podrían haber existido sin aportes de capital de riesgo o se habrían desarrollado mucho más lentamente.

- Para las empresas de alto potencial de crecimiento, los aportes de capital de riesgo son mucho más adecuados que el préstamo bancario.

- Durante varios años, estas empresas no podrían haber atendido la devolución de un crédito bancario. 
- Se ha creado un promedio de 46 empleos adicionales por las empresas que han recibido aportes de capital de riesgo.

Para incrementar sus operaciones, las empresas necesariamente requieren de mayores recursos o, en su defecto, necesitan alcanzar mayores niveles de eficiencia, sin que por ello pierdan eficacia, sino que más bien deberán preocuparse principalmente por la consecución de mayor eficacia en el proceso de optimización de sus recursos, lo cual puede ser visto como un ideal utópico por quienes ven este aspecto como irrealizable. Nos atrevemos a sostener que esto último es un pensamiento pesimista de quienes no han explotado todas sus posibilidades y potencialidades, o, lo que es peor, no conocen las que tienen ni cómo pueden disponer de ellas.

\section{La necesidad de cambio}

Es un hecho que lo único constante es el cambio, y para quienes así lo asumen, cada día es un reto que al finalizar representa la recompensa de haber superado la prueba, por más similar que resultara al pasado (24).
Es de por sí y hasta cierto punto obvio que las empresas no pueden quedarse sin aplicar cambios de manera incesante, pero también es muy cierto que los cambios generan inestabilidad para los agentes empresariales. Incurrir en cambios y perder el control de estos (desconocer su principio, rumbo y fin) significa la debacle inminente y probablemente irreversible, y seguramente si no se toman medidas racionales y urgentes, la quiebra estará prontamente en la agenda.

\section{El problema de la calidad y el empowerment}

El problema de crecer, en el aspecto del personal que la empresa pueda contratar, y también respecto al personal que ya se encuentra incorporado a las operaciones cotidianas que desarrolla, radica en el logro y mejoramiento de la calidad, pues adquirir una filosofía de trabajo y más aún una filosofía de vida es en extremo una cuestión de gran complejidad. Por ello es de vital importancia efectuar un proceso de inducción del personal a la empresa, mucho antes de que se incorpore a los procesos de producción de los bienes y servicios a los que dicha empresa se vea enfocada.

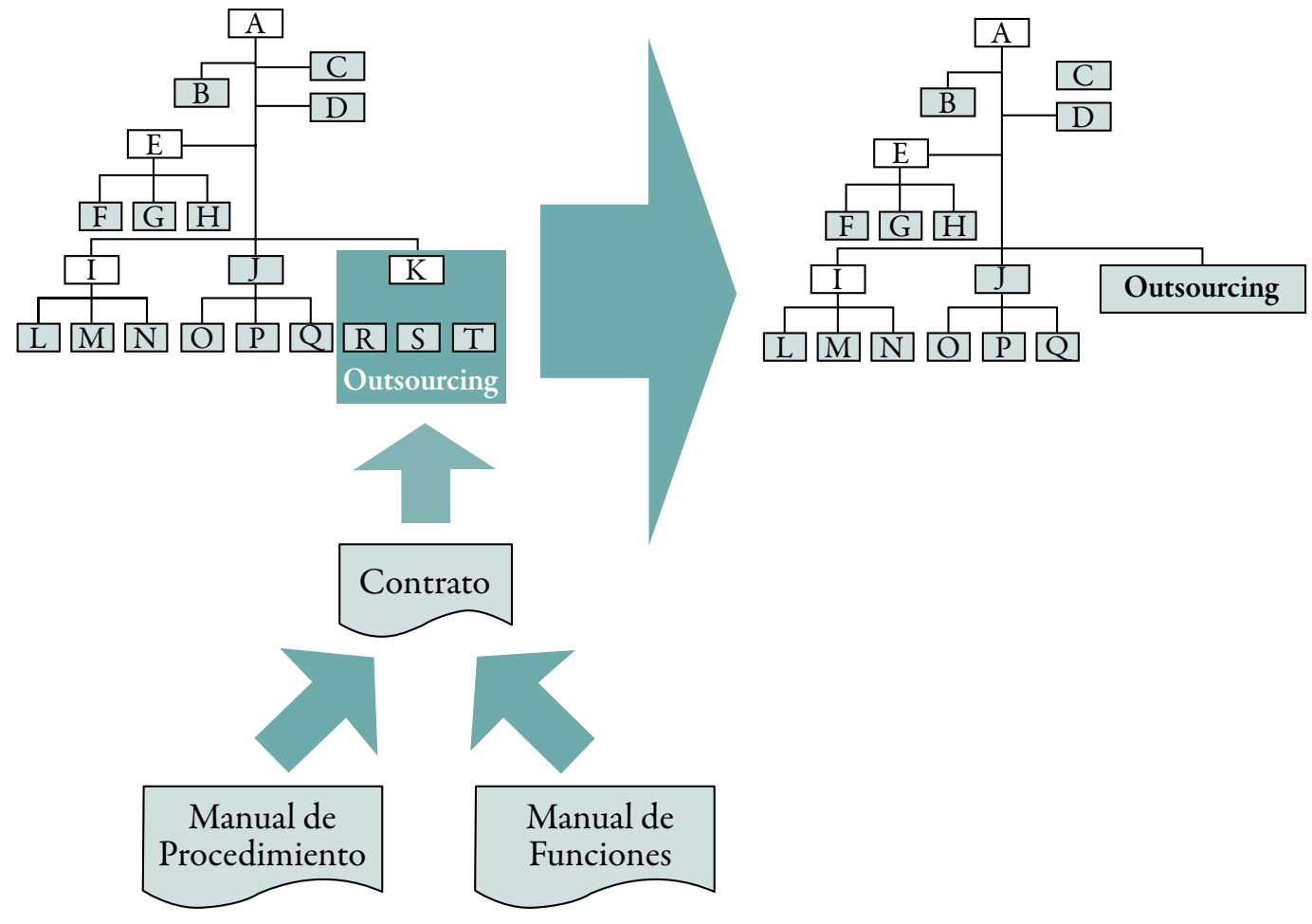


La implementación de un empowerment en el proceso de lograr la calidad debe ser complementada con un plan muy bien elaborado, dirigido a lograr un compromiso constante del personal así como a cumplir con los objetivos empresariales, y en forma paralela planificar la autonomía de los órganos o unidades estratégicas de negocio (UEN), todo con el fin de explotar la potencialidad del empowerment.

El problema de la carencia de recursos, el crecimiento y el outsourcing

Si se logra la independencia de ciertas UEN y se fortalece la capacidad de toma de decisiones de su ámbito de acción, en el cual han desarrollado gran especialización, están preparadas para formar una empresa autónoma dentro de la figura de outsourcing. Así, puede adquirir ciertos activos, maquinaria y equipos que no sean puntales del núcleo del negocio, con lo que la empresa estaría en la posibilidad de recuperar fondos para ser aplicados a otras áreas neurálgicas, sin perder operatividad en la separación de la mencionada UEN.

\section{Caso práctico}

En nuestra localidad, en una entidad financiera, existía una área de mantenimiento de computadoras, la misma que hacía uso de cierta infraestructura, maquinaria y equipo para desarrollar sus funciones. En este caso, un número de trabajadores de esta área recibió la propuesta de adquirir todos estos recursos a monto financiado por la misma institución, pero con la inyección de capital por parte de estos, quienes optaron por renunciar y utilizar los fondos de su compensación por tiempo de servicios para cubrir el monto requerido.

La institución financiera obtuvo una área con especialistas autocontrolados (outsourcing) que de forma nativa conocían dos aspectos fundamentales para lograr un nivel muy alto de integración, eficiencia y eficacia:
- La calidad de sus procesos y servicios.

- Conocimiento del negocio y de los pormenores de su participación.

\section{CONCLUSIONES}

El empowerment en el proceso de la estrategia de crecimiento debe emplearse con el fin de lograr unidades estratégicas de negocio autónomas y especializadas, con una idea clara del negocio y de la calidad.

El outsourcing se debe aplicar a las unidades estratégicas de negocio con un ámbito de aplicación que no sea el núcleo del negocio, asumiendo como parámetros de definición del contrato las funciones y procedimientos integrados en el proceso de negocio.

Los recursos recuperados en el proceso de creación de nuevas empresas en la figura del outsourcing deben ser empleados para acotar las estrategias de crecimiento.

\section{REFERENCIAS BIBLIOGRÁFICAS}

1. Yohann Johnson. Empowerment. bttp://www.gestiopolis.com/recursos/documentos/fulldocs/rrhb/empowerment.htm

2. Alexander Cifuentes R. Empowerment. bttp://www.monografias.com/trabajos 11 power/power.shtm

3. Improven Consultores, Pascual y Genís. Empowerment... ¿una necesidad en el entorno actual? bttp://www.improven-consultores.com, pdflempowerment.pdf

4. Gestiopolis. bttp://www.gestiopolis.com/recursos/documentos/fulldocs/rrhb/empwuch.pdf

5. Lesneika Botín. El empowerment y toma de decisiones. bttp://www.gestiopolis.com/recursos4/docs/ger/empowerment.htm 
6. Francesco di Castri. La hora del empowerment. El poder del conocimiento.

bttp://www.revista-ambiente.com.ar imagenes/95/empowerment.pdf

7. José Ramón Sida. Sociedad mexicana para el desarrollo de calidad total, S.C.

8. Bill Ginnodo. The Power of Empowerment, What the experts say and 16 actionable case studies.

9. Peter Block. The empowered manager. Positive political skills at work. Jossey-Bass Publishers, San Francisco Oxford, 2001, U.S.A.

10. www.fundameca.org.mx

11. Pablo Lara Navarra y José Ángel Martínez. User outsourcing documental:organización del futuro.

bttp://eprints.rclis.org/archive/00006968/ 01/2000-FESABID-outsourcing.pdf

12. Wikipedia. Subcontratación.

http://es.wikipedia.org/wiki/Subcontrataci $\% \mathrm{C} 3 \% \mathrm{~B} 3 \mathrm{n}$

13. Ruddy Fernández. Outsourcing estrategia empresarial del presente y futuro. bttp://www. monografias.com/trabajos10/outso/outso.shtm

14. Carlos López. El outsourcing o la tercerización. bttp://www.gestiopolis.com/canales/gerencial/articulos/no\%209/outsourcing.htm

15. Computación UnixSupport, C.A. Outsourcing. bttp://www.unixsup.com/outsourcing/index. btmo
16. Nidia Aragón. Reingeniería (BPR: Business Process Reenginering). bttp://www.gestiopolis. com/recursos4/docs/ger/reingenieria.htm

17. Ruddy Fernández. Outsourcing, estrategia empresarial del presente y futuro.

bttp://www.monografias.com/trabajos10/outso/outso.shtml

18. Gustavo Rubinsztejn. Tercerización: ventajas, desventajas y criterios para su adopción.

19. Improven Consultores. El outsourcing como opción estratégica.

www.improven-consultores.com/pdf/outsourcing.pdf

20. Sacar la operación y retener la inteligencia.

bttp://www.idg.es/comunicaciones/pdf/ outsourcing.pdf

21. Carlos López. El outsourcing o la tercerización. bttp://www.gestiopolis.com/canales/gerencial/articulos/no\%209/outsourcing.htm

22. Francisco J. Forcadell. El crecimiento empresarial desde el enfoque basado en los recursos. Hacia un modelo integrador.

bttp://www3.uva.es/empresa/uploads dt_12_04.pdf

23. Blanca Revenga. Factores condicionantes del tamaño y del crecimiento empresarial. bttp:/ www.ipyme.org/NR/rdonlyres/8818AC092 D464EB498B9745A2ADD889E/0/6MIC Egopherselephantsandgazelles.pdf

24. Félix Óscar Socorro Márquez. ¡Probibido innovar! iii: Lo unico constante es el cambio. bttp://www.gestiopolis.com/canales/emprendedora/articulos/59/probiinno.htm 